\title{
Compressibilidade de um Argissolo sob plantio direto escarificado e compactado
}

\author{
Compressibility of a Paleudalf chisel plough and compacted under no-tillage
}

\author{
Cláudia Liane Rodrigues de Lima ${ }^{1}$ Dalvan Jose Reinert ${ }^{2}$ \\ José Miguel Reichert ${ }^{2}$ Luis Eduardo Akiyoshi Sanches Suzuki ${ }^{3}$
}

\section{RESUMO}

A compressibilidade do solo é dependente do tipo, da intensidade, da freqüência da força aplicada por máquinas agrícolas e dos sistemas de manejo adotados. $O$ objetivo deste estudo foi avaliar o efeito da intensidade do tráfego de máquinas agrícolas na compressibilidade de um Argissolo Vermelho distrófico arênico sob plantio direto escarificado e compactado. Foram testados os tratamentos: $P D=$ plantio direto desde $o$ ano de 1989; PDE1 = plantio direto escarificado em dezembro de 2002 e fevereiro de 2004; PDE2 = plantio direto escarificado em dezembro de 2004; PDC1, PDC2 e PDC3 = plantio direto com quatro passadas de máquina com massa total de $10 \mathrm{Mg}$ em dezembro, respectivamente para os anos agrícolas 2001/ 2002, 2002/2003 e 2001/2002 - 2002/2003. Amostras com estrutura de solo preservada, na camada de 0,08-0,13m, foram utilizadas para avaliar os parâmetros compressivos do solo. No sistema de plantio direto sob compactação adicional, foram obtidos maiores e menores valores, respectivamente, de densidade e índice de compressão do solo. A densidade e o índice de compressão do solo são parâmetros consistentes $e$ sensíveis para detectar alterações na estrutura do solo. $O$ modelo não-linear (MCNABB \& BOERSMA, 1993) é uma ferramenta potencial para determinação da densidade do solo sob plantio direto, a partir da densidade inicial e das pressões aplicadas ao solo.

Palavras-chave: compactação do solo, pressão de preconsolidação, índice de compressão, curva de compressão.

\section{ABSTRACT}

Soil compressibility depends on the type, intensity and frequency of the load applied by agricultural machinery and on soil management. This study was aimed at evaluating the effect of traffic of agricultural machines on the compressibility of a Paleudalf chisel plough and compacted under no-tillage. In an area under no tillage since 1989, the following treatments were used for sampling: $P D=$ no tillage since 1989; PDE1 = no tillage plus chisel plough in december the year 2002 and february 2004; PDE2 = no tillage plus chisel plough in december 2004; PDC1, PDC2 and PDC3 = no tillage plus four passes of a machine of $10 \mathrm{Mg}$ in December, respectively to the agricultural years of 2001/2002, 2002/2003 and 2001/2002 - 2002/2003. Soil cores were collected at 0.08$0.13 \mathrm{~m}$ depth to evaluate the compressive parameters of soil. In the no tillage under additional compaction higher and lower values were obtained respectively of bulk density and soil compression index. The bulk density and the soil compression index are concistant and sensitive parameters to detect alterations in the soil structure. The non linear model (MCNABB \& BOERSMA, 1993) is a potential tool to determine the bulk density of soils under no tillage, starting from the initial bulk density and the loads to be applied in the soil.

Key words: soil compaction, preconsolidation pressure, compression index, compression curve.

\section{INTRODUÇÃo}

A caracterização do processo de compressão contribui para definir ações do manejo que evitem ou minimizem a degradação da qualidade física dos solos. Indicadores relacionados à compressibilidade, obtidos a partir da curva de compressão uniaxial, têm sido reconhecidos e utilizados para estabelecer condições adequadas ao desenvolvimento das plantas (MOSADDEGHI et al, 2003). A pressão de preconsolidação é um indicador da

${ }^{1}$ Departamento de Solos, Centro de Ciências Rurais (CCR), Universidade Federal de Santa Maria (UFSM), 97105-900, Santa Maria, RS, Brasil. E-mail: clrlima@yahoo.com.br. Autor para correspondência.

${ }^{2}$ Departamento de Solos, CCR, UFSM, 97105-900, Santa Maria, RS, Brasil. E-mail: reinert@ccr.ufsm.br.

${ }^{3}$ Departamento de Ciências Florestais, CCR, UFSM, 97105-900, Santa Maria, RS, Brasil. E-mail: suzuki@mail.ufsm.br. 
pressão máxima exercida (SALIRE et al., 1994) e da capacidade de suporte de carga com vistas à prevenção da compactação adicional do solo (LEBERT \& HORN, 1991; DIAS JUNIOR \& PIERCE, 1995). O índice de compressão representa a susceptibilidade do solo à compactação (LARSON et al., 1980; SILVAet al., 2000).

A modelagem tem apresentado potencialidade para o entendimento do processo de compressão. Estudos sobre a reta de compressão virgem para diferentes solos e teores de água foram desenvolvidos por LARSON et al. (1980). Os autores apresentaram curvas de compressão uniaxial e avaliaram a susceptibilidade à compactação de oito tipos de solos com variação no teor de água e argila. Um modelo nãolinear para determinar a pressão máxima que o solo pode suportar sem sofrer compactação adicional, baseando-se em diferentes teores de água, foi desenvolvido por DIAS JUNIOR \& PIERCE (1995). KONDO (1998), utilizando o mesmo modelo, em Latossolos, demonstrou que a compressibilidade é influenciada por diferentes sistemas de manejo. KONDO \& DIAS JUNIOR (1999) avaliaram a pressão de preconsolidação em Latossolos sob diferentes teores de água do solo sob cultura anual, mata e pastagem. SILVA et al. (2000) testaram modelos em dois tipos de solos e manejos para estimar a pressão de preconsolidação e o índice de compressão. Os autores concluíram que existe dependência da densidade inicial e dos graus de saturação de água na compressibilidade do solo, corroborando os resultados de KONDO \& DIAS JUNIOR (1999). IMHOFF et al. (2004), empregando amostras de Latossolos, com ampla variação de textura em uma topossequiência sob cultivo de cana de açúcar, apresentaram um modelo que incorpora o efeito do teor de água, argila e densidade na pressão de preconsolidação do solo.

Em sistemas mecanizados, o conhecimento dos valores da capacidade de suporte de carga e da susceptibilidade à compactação pode auxiliar na escolha de máquinas que exerçam menores pressões e na redução do impacto causado à qualidade estrutural de solos agrícolas (KONDO \& DIAS JUNIOR, 1999; MOSADDEGHI et al., 2003). Nesse sentido, testou-se a hipótese de a compressibilidade do solo ser alterada por sistemas de manejo e intensidades de tráfego. $\mathrm{O}$ objetivo deste trabalho foi avaliar o efeito da intensidade do tráfego de máquinas agrícolas na compressibilidade de um Argissolo Vermelho distrófico arênico sob plantio direto escarificado e compactado.

\section{MATERIAL E MÉTODOS}

Em uma área $\left(1512 \mathrm{~m}^{2}\right)$ pertencente ao Departamento de Solos da Universidade Federal de
Santa Maria, UFSM, RS (Latitude de 29 45' S; Longitude de $53^{\circ} 42^{\prime}$ W; $95 \mathrm{~m}$ ), foram utilizados três experimentos (A, B, C), delineados em blocos casualizados com quatro repetições, totalizando 36 parcelas. O clima da região é caracterizado como Cfa (subtropical úmido sem estiagens), de acordo com a classificação de Köppen, sendo a temperatura média do mês mais quente superior a $22^{\circ} \mathrm{C}$ e a do mês mais frio entre $-3^{\circ} \mathrm{C}$ e $18^{\circ} \mathrm{C}$ (MORENO, 1961). O solo foi classificado como Argissolo Vermelho distrófico arênico (EMBRAPA, 1999), de textura superficial franco arenosa $\left(81 \mathrm{~g} \mathrm{~kg}^{-1}\right.$ de argila, $291 \mathrm{~g} \mathrm{~kg}^{-1}$ de silte e $628 \mathrm{~g} \mathrm{~kg}^{-1}$ de areia).

Em área sob plantio direto desde 1989, foram testados os tratamentos: $\mathrm{PD}=$ plantio direto desde $\mathrm{o}$ ano de 1989; PDE1 = plantio direto escarificado em dezembro de 2002 e fevereiro de 2004; PDE2 = plantio direto escarificado em dezembro de 2004; PDC1, PDC2 e PDC3 = plantio direto com quatro passadas de máquina com massa total de aproximadamente $10 \mathrm{Mg}$ em dezembro, respectivamente para os anos agrícolas 2001/2002, 2002/2003 e 2001/2002 - 2002/2003. Foram comparados no experimento A, os tratamentos PDC3, PD e PDE2; no experimento B, os tratamentos PDC1, $\mathrm{PD}$ e PDE1; e no experimento $\mathrm{C}$, os tratamentos PDC2, PD e PDE2.

O processo de compactação adicional e as especificações das máquinas utilizadas podem ser obtidas em STRECK (2003) e COLLARES (2005). A escarificação foi feita até a profundidade de $0,20 \mathrm{~m}$, com um escarificador de quatro hastes espaçadas em aproximadamente $0,20 \mathrm{~m}$.

Em maio de 2005, foi feita a amostragem com estrutura de solo preservada na camada de 0,08 a $0,13 \mathrm{~m}$, com cilindros metálicos $(0,025 \mathrm{~m}$ de altura e $0,0610 \mathrm{~m}$ de diâmetro interno). As amostras foram saturadas em água por 24 horas e equilibradas a $33 \mathrm{kPa}$ em câmaras de pressão (KLUTE, 1986). Após o equilíbrio, foram submetidas a testes de compressão uniaxial utilizandose um consolidômetro S-450 Terraload (Durham GeoInterprises). $\mathrm{O}$ ensaio consistiu na aplicação sucessiva e contínua de pressões $(12,5,25,50,100,200,400,800$ e $1.600 \mathrm{kPa})$, por cinco minutos, período suficiente para atingir $99 \%$ da deformação máxima (SILVA et al., 2000). Após esse ensaio, as amostras foram colocadas em estufa para secagem a $105^{\circ} \mathrm{C}$, por 24 horas, e, a partir da massa seca e do volume do solo, foi determinada a densidade (Ds) (BLAKE \& HARTGE, 1986) para cada pressão aplicada. Com base nesses valores, foi feita a curva de compressão uniaxial (medida) para cada amostra, que consistiu na relação entre o logaritmo da pressão aplicada $(\log \sigma)($ eixo x) e a Ds (eixo y). Por meio de ajuste manual (método 2) e com auxílio do 
programa computacional Compress (REINERT et al., 2003), foram obtidos os valores de pressão de preconsolidação $\left(\sigma_{\mathrm{p}}\right)$ e do índice de compressão do

solo $\left.I C=\frac{d D s}{d \log \sigma}\right)($ CASAGRANDE, 1936). Em

cada experimento, os valores de Ds, $\sigma_{\mathrm{p}}$ e IC foram submetidos à análise de variância e comparados pelo teste que considera a diferença mínima significativa a $5 \%$ (DMS).

Paralelamente, o modelo não-linear proposto por MCNABB \& BOERSMA (1993) foi utilizado para ajustar as curvas de compressão medidas e modelar o processo de compactação. Para isso, utilizouse a equação:

$\ln D s=\ln \left(D s_{0} \delta\right)-\left(a+b \sigma+c \delta_{c}\right) \times\left(1-e^{-d \sigma}\right)$ onde: In Ds é o logaritmo natural da densidade do solo $\left(\mathrm{Mg} \mathrm{m}^{-3}\right), \mathrm{Ds}_{0}$ é a densidade estimada a $0 \mathrm{kPa}\left(\mathrm{Mg} \mathrm{m}^{-3}\right)$, Dsi é a densidade inicial $\left(\mathrm{Mg} \mathrm{m}^{-3}\right)$, Dsi ${ }_{\text {média }}$ é a densidade inicial média $\left(\mathrm{Mg} \mathrm{m}^{-3}\right), \delta_{i}=\frac{D s_{i}}{D s i_{\text {média }}}, \sigma$ é a pressão, é a pressão aplicada $(\mathrm{kPa}), \delta_{c}=\left(\delta_{i}-1\right) \times D s_{0}(\mathrm{Mg}$ $\mathrm{m}^{-3}$ ) e a, b e c são parâmetros do modelo que descrevem a forma da curva de compressão.

\section{RESULTADOS E DISCUSSÃO}

Níveis de compactação diferenciados condicionaram variação nos valores de densidade inicial (Ds) (Tabela 1). A figura 1 mostra o comportamento dos parâmetros físicos do solo empregados no presente estudo. Houve diferenças de Ds entre os sistemas de manejo nos experimentos A e C (Figura 1). O sistema plantio direto que recebeu compactação adicional (PDC2 e PDC3) apresentou os maiores valores de Ds, não diferindo do solo sob PD (experimento A) e do sistema que recebeu escarificação (PDE2) (experimento C). O efeito significativo da compactação sobre a Ds também foi observado por SECCO et al. (2004) em um Latossolo sob plantio direto.

Considerando que tratores agrícolas exercem ao solo pressões de 70 a 350kPa (SOANE, 1986), o intervalo de variação da pressão de preconsolidação dos três experimentos $\left(73<\sigma_{\mathrm{p}}<288 \mathrm{kPa}\right)$ (Tabela 1$)$ concorda com valores de pressões de máquinas agrícolas exercidas ao solo.

A $\sigma_{p}$, que representa a capacidade de suporte de carga do solo, não apresentou tendência em ser influenciada por níveis de compactação (Figura 1), corroborando os resultados apresentados por SILVA et al. (2002a), para um Latossolo Vermelho distrófico. Observou-se que a $\sigma_{\mathrm{p}}$ não foi dependente da densidade do solo ( $\mathrm{P}<0,9480)$, corroborando com MOSADDEGHI et al. (2003). Porém, há indicações de que a $\sigma_{\mathrm{p}}$ seja influenciada pela Ds (SILVA et al., 2002a; IMHOFF et al., 2004). SALIRE et al. (1994) e ALEXANDROU \& EARL (1998) apresentaram uma relação linear e positiva entre a $\sigma_{\mathrm{p}}$ e a Ds, ou seja, menores valores de densidade estiveram associados à menor capacidade de suporte de carga. SILVA et al. (2002a), considerando intervalos diferenciados de Ds, observaram menores valores médios de $\sigma_{\mathrm{p}}$ em um

Tabela 1 - Parâmetros das variáveis analisadas.

\begin{tabular}{|c|c|c|c|c|c|}
\hline Variáveis ${ }^{(1)}$ & Média & Desvio padrão & Mínimo & Máximo & $\mathrm{CV}(\%)$ \\
\hline & \multicolumn{5}{|c|}{ Experimento A } \\
\hline $\mathrm{Ug}$ & 0,096 & 0,006 & 0,085 & 0,104 & 6,852 \\
\hline Ds & 1,61 & 0,09 & 1,41 & 1,71 & 5,66 \\
\hline$\sigma_{\mathrm{p}}$ & 151,7 & 52,6 & 73,4 & 288,2 & 34,7 \\
\hline \multirow[t]{2}{*}{ IC } & 0,190 & 0,059 & 0,130 & 0,340 & 31,419 \\
\hline & \multicolumn{5}{|c|}{ Experimento B } \\
\hline $\mathrm{Ug}$ & 0,093 & 0,005 & 0,086 & 0,099 & 4,938 \\
\hline Ds & 1,62 & 0,07 & 1,51 & 1,74 & 4,53 \\
\hline$\sigma_{\mathrm{p}}$ & 149,6 & 31,0 & 103,8 & 195,9 & 20,7 \\
\hline \multirow[t]{2}{*}{ IC } & 0,183 & 0,057 & 0,120 & 0,290 & 31,215 \\
\hline & \multicolumn{5}{|c|}{ Experimento C } \\
\hline Ug & 0,088 & 0,006 & 0,077 & 0,094 & 6,472 \\
\hline Ds & 1,61 & 0,09 & 1,48 & 1,75 & 5,94 \\
\hline$\sigma_{\mathrm{p}}$ & 153,1 & 29,7 & 121,2 & 203,9 & 19,4 \\
\hline IC & 0,181 & 0,0816 & 0,090 & 0,300 & 45,063 \\
\hline
\end{tabular}

\footnotetext{
${ }^{(1)} \mathrm{Ug}=$ umidade gravimétrica $\left(\mathrm{kg} \mathrm{kg}^{-1}\right) ; \mathrm{Ds}=$ densidade inicial do solo $\left(\mathrm{Mg} \mathrm{m}^{-3}\right) ; \sigma_{\mathrm{p}}=$ pressão de preconsolidação $(\mathrm{kPa})$ e IC = índice de
} compressão do solo. 


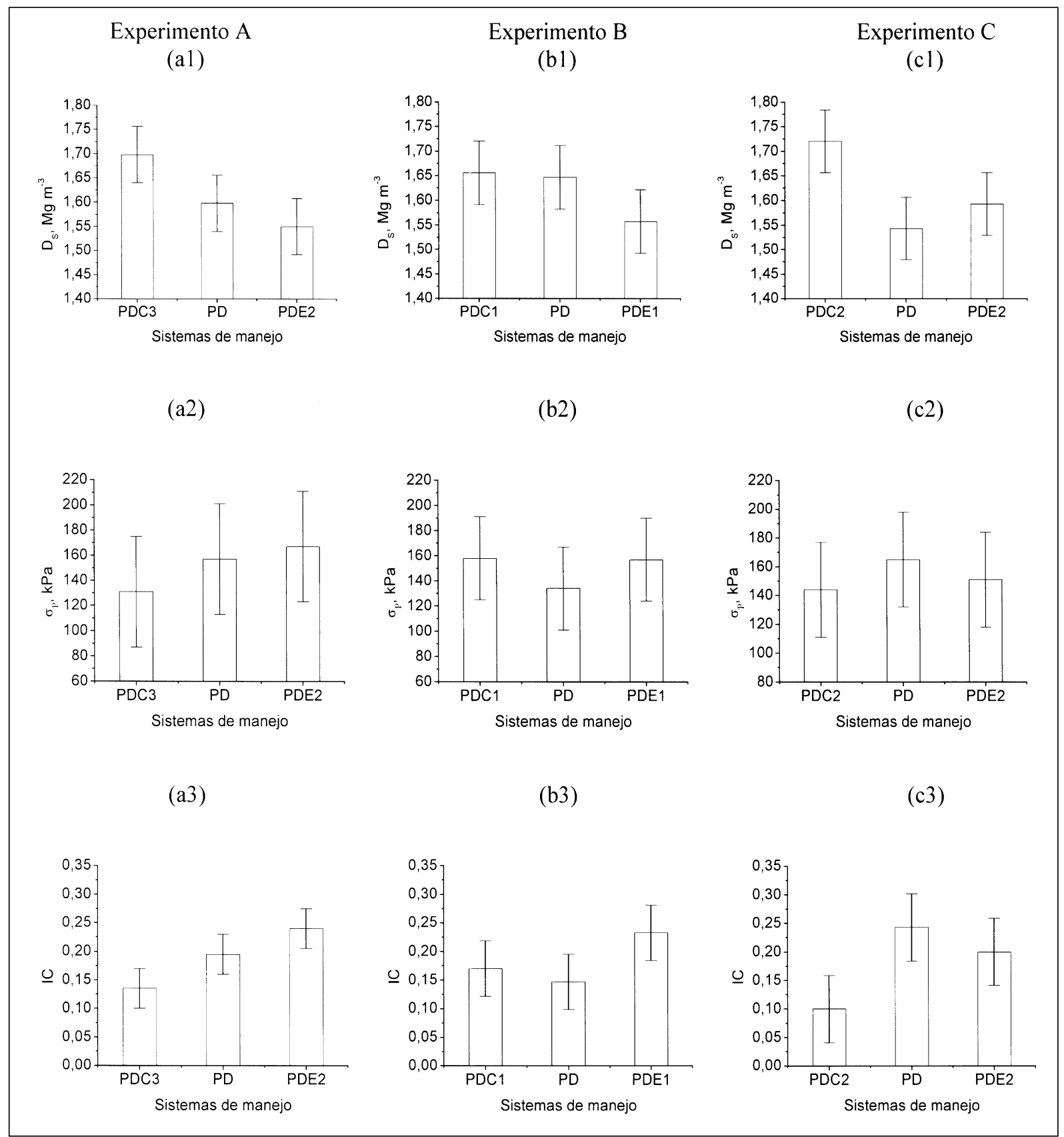

Figura 1 - Densidade inicial (Ds), capacidade de suporte de carga $\left(\sigma_{\mathrm{p}}\right)$ e índice de compressão (IC) de um Argissolo Vermelho distrófico arênico sob sistemas de manejo ${ }^{1}$ nos experimentos (a1, a2, a3) A, (b1, b2, b3) B e (c1, c2, c3) C.

${ }^{1} \mathrm{PD}$ = plantio direto desde o ano de 1989; SDE1 = plantio direto escarificado em fevereiro de 2002 e dezembro de 2004; SDE2 = plantio direto escarificado em dezembro de 2004; SDC1 = plantio direto com quatro passadas de máquina de $10 \mathrm{Mg}$ em dezembro do ano agrícola 2001/2002; SDC2 = plantio direto com quatro passadas de máquina de $10 \mathrm{Mg}$ em dezembro do ano agrícola 2002/2003; SDC3 = plantio direto com quatro passadas de máquina de $10 \mathrm{Mg}$ em dezembro do ano agrícola 2001/ 2002 e 2002/2003.

mesmo tipo de solo sob plantio direto. Esse fato está associado ao teor de umidade ou ao grau de saturação das amostras utilizadas (50-80\%) em relação ao deste estudo (31-50\%). SILVA et al. (2002b) observaram valores médios semelhantes de $\sigma_{\mathrm{p}}$ quando utilizaram amostras com grau de saturação na faixa de 31 a $60 \%$, para um mesmo tipo de solo e sistema de manejo.

O valor médio de índice de compressão foi semelhante ao de LIMA et al. (2004), fato que pode ser explicado por esses solos apresentarem textura semelhante (Tabela 1). Observaram-se diferenças

Ciência Rural, v.36, n.6, nov-dez, 2006. 
significativas de IC nos experimentos Ae C (Figura 1). O IC do PD e do PDE2 foram semelhantes (experimentos A e C), ou seja, considerando que o IC seja a facilidade com que um determinado solo aumenta de $\mathrm{D}_{\mathrm{S}}$ quando submetido a um aumento de pressão aplicada e umidade (SMITH et al., 1997), menores níveis de compactação condicionarão maior susceptibilidade do solo à degradação estrutural nesses sistemas.

Relações negativas entre IC e Ds foram apresentadas por SMITH et al. (1997). No presente estudo, valores de IC entre os sistemas de manejo também estiveram relacionados negativamente à Ds no experimento $\mathrm{A}\left(\mathrm{F}=104,41 ; \mathrm{P}<0,0001 ; \mathrm{R}^{2}=0,90\right)$, B $\left(\mathrm{F}=32,30 ; \mathrm{P}<0,0007 ; \mathrm{R}^{2}=0,80\right)$ e $\mathrm{C}(\mathrm{F}=205,19 ; \mathrm{P}<$ 0,$\left.0001 ; \mathrm{R}^{2}=0,96\right)$.

Pelo conhecimento do índice de compressão, é possível prever mudanças na relação ar-água-matriz quando determinada pressão externa é exercida ao solo e, por sua vez, condições mais adequadas ao crescimento das plantas (GUPTA \& ALLMARAS, 1987). De modo geral, o sistema que recebeu compactação adicional apresentou a menor susceptibilidade do solo à compactação em comparação aos demais tratamentos.

A partir dos valores medidos e dos parâmetros do modelo de MCNABB \& BOERSMA (1993) (Tabela 2), foram construídas, respectivamente, as curvas de compressão medidas e estimadas para cada tratamento e experimento (Figura 2). As curvas de compressão refletiram diferenças no processo de compactação dos solos. Apesar de no experimento B não serem observadas diferenças nos valores de densidade inicial, o solo que recebeu escarificação (PDE1) apresentou tendência na diminuição de Ds em

Tabela 2 - Parâmetros do modelo $\ln D s=\ln \left(D s_{0} \delta\right)-\left(a+b \sigma+c \delta_{c}\right) \times\left(1-e^{-d \sigma}\right)$ de um Argissolo Vermelho distrófico arênico sob sistemas de manejo ${ }^{1}$ dos experimentos $\mathrm{A}, \mathrm{B}$ e $\mathrm{C}$.

\begin{tabular}{|c|c|c|c|c|c|c|}
\hline \multirow[t]{2}{*}{ Parâmetros } & Valor estimado & Erro Padrão & Valor estimado & Erro Padrão & Valor estimado & Erro Padrão \\
\hline & \multicolumn{2}{|c|}{ Experimento A } & \multicolumn{2}{|c|}{ Experimento B } & \multicolumn{2}{|l|}{ Experimento $\mathrm{C}$} \\
\hline & \multicolumn{2}{|c|}{$\mathrm{PD}^{1}$} & \multicolumn{2}{|c|}{$\mathrm{PD}^{4}$} & \multicolumn{2}{|c|}{$\mathrm{PD}^{7}$} \\
\hline$D s_{0}$ & 1,594 & 0,001 & 1,645 & 0,002 & 1,536 & 0,003 \\
\hline $\mathrm{a}$ & $-0,072$ & 0,005 & $-0,049$ & 0,004 & $-0,189$ & 0,047 \\
\hline $\mathrm{b}$ & $-0,047$ & 0,004 & $-0,046$ & 0,004 & $-0,002$ & 0,022 \\
\hline $\mathrm{c}$ & 4,472 & 0,516 & 6,681 & 1,052 & 1,509 & 0,442 \\
\hline \multirow[t]{2}{*}{ d } & 0,206 & 0,045 & 1,325 & 0,254 & 0,542 & 0,077 \\
\hline & \multicolumn{2}{|c|}{$\mathrm{PDE}^{2}$} & \multicolumn{2}{|c|}{$\mathrm{PDE}^{5}$} & \multicolumn{2}{|c|}{$\mathrm{PDE} 2^{8}$} \\
\hline$D s_{0}$ & 1.546 & 0,003 & 1,555 & 0,004 & 1,589 & 0,008 \\
\hline a & $-0,126$ & 0,011 & $-0,109$ & 0,028 & $-0,163$ & 0,120 \\
\hline $\mathrm{b}$ & $-0,039$ & 0,007 & $-0,041$ & 0,017 & $-0,001$ & 0,059 \\
\hline $\mathrm{c}$ & 3,104 & 0,352 & 2,797 & 0,900 & 1,671 & 1,488 \\
\hline \multirow[t]{2}{*}{ d } & 0,593 & 0,026 & 0,472 & 0,067 & 0,498 & 0,215 \\
\hline & \multicolumn{2}{|c|}{$\mathrm{PDC}^{3}$} & \multicolumn{2}{|c|}{$\mathrm{PDC}^{6}$} & \multicolumn{2}{|c|}{$\mathrm{PDC}^{9}$} \\
\hline$D s_{0}$ & 1,695 & 0,005 & 1,649 & 0,002 & 1,716 & 0,002 \\
\hline a & $-0,064$ & 0,01 & $-0,161$ & 0,049 & $-0,038$ & 0,004 \\
\hline $\mathrm{b}$ & $-0,033$ & 0,008 & 0,007 & 0,021 & $-0,028$ & 0,003 \\
\hline $\mathrm{c}$ & 5,311 & 1,579 & 1,234 & 0,426 & 5,668 & 0,937 \\
\hline $\mathrm{d}$ & $-0,102$ & 0,274 & 0,416 & 0,075 & 0,119 & 0,038 \\
\hline
\end{tabular}

ln Ds é o logaritmo natural da densidade do solo $\left(\mathrm{Mg} \mathrm{m}^{-3}\right), \mathrm{Ds}_{0}$ é a densidade estimada a $0 \mathrm{kPa}\left(\mathrm{Mg} \mathrm{m}^{-3}\right)$, Dsi é a densidade inicial $\left(\mathrm{Mg}^{-3}\right)$, Dsi média: densidade inicial média $\left(\mathrm{Mg} \mathrm{m}^{-3}\right), \delta_{i}=\frac{D s_{i}}{D s i_{\text {média }}}, \sigma$ é a pressão aplicada $(\mathrm{kPa}), \delta_{c}=\left(\delta_{i}-1\right) \times D s_{0}\left(\mathrm{Mg} \mathrm{m}^{-3}\right)$ e a, b e c são parâmetros do modelo que descrevem a forma da curva de compressão.

${ }^{1} \mathrm{PD}=$ plantio direto desde o ano de $1989\left(\mathrm{~F}=10445,3 ; \mathrm{P}<0,0001 ; \mathrm{R}^{2}=0,99\right) ;{ }^{2} \mathrm{PDE} 2=$ plantio direto escarificado em dezembro de $2004(\mathrm{~F}$ $\left.=6154,19 ; \mathrm{P}<0,0001 ; \mathrm{R}^{2}=0,99\right) \mathrm{e}^{3} \mathrm{PDC} 3=$ plantio direto com quatro passadas de máquina de $10 \mathrm{Mg}$ em dezembro no ano agrícola

2001/2002 e 2002/2003 ( $\left.\mathrm{F}=884,65 ; \mathrm{P}<0,0001 ; \mathrm{R}^{2}=0,96\right) .{ }^{4} \mathrm{PD}=$ plantio direto desde $\mathrm{o}$ ano de $1989\left(\mathrm{~F}=3377,60 ; \mathrm{P}<0,0001 ; \mathrm{R}^{2}=0,99\right)$; ${ }^{5} \mathrm{PDE} 1$ = plantio direto escarificado em dezembro de 2002 e $2004\left(\mathrm{~F}=1987,09 ; \mathrm{P}<0,0001 ; \mathrm{R}^{2}=0,99\right) ;{ }^{6} \mathrm{PDC} 1=$ plantio direto com quatro passadas de máquina de $10 \mathrm{Mg}$ em dezembro no ano agrícola 2001/2002 (F = 5299,03; $\left.\mathrm{P}<0,0001 ; \mathrm{R}^{2}=0,99\right)$. ${ }^{7} \mathrm{PD}=$ plantio direto desde $\mathrm{o}$ ano de $1989\left(\mathrm{~F}=4312,60 ; \mathrm{P}<0,0001 ; \mathrm{R}^{2}=0,99\right) ;{ }^{8} \mathrm{PDE} 2=$ plantio direto escarificado em dezembro de $2004\left(\mathrm{~F}=457,58 ; \mathrm{P}<0,0001 ; \mathrm{R}^{2}=\right.$ $0,95)$ e ${ }^{9} \mathrm{PDC} 2$ = plantio direto com quatro passadas de máquina de $10 \mathrm{Mg}$ em dezembro no ano agrícola 2002/2003 (F = 4065,64; $\mathrm{P}<$ 0,$\left.0001 ; \mathrm{R}^{2}=0,99\right)$.

Ciência Rural, v.36, n.6, nov-dez, 2006. 


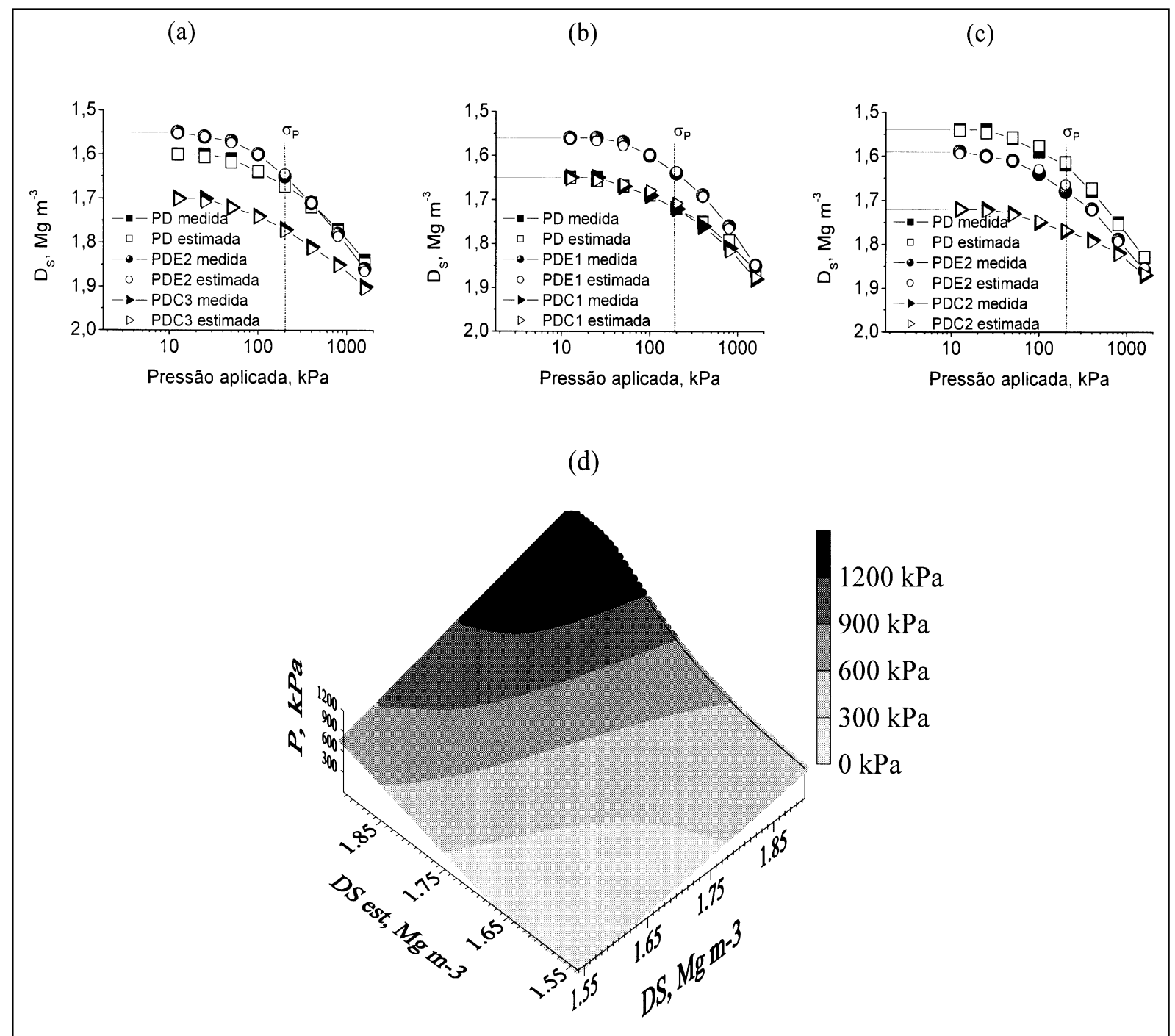

Figura 2 - Curvas de compressão uniaxial medidas e estimadas pelo modelo de MCNABB \& BOERSMA (1993) de um Argissolo Vermelho distrófico arênico sob sistemas de manejo ${ }^{1}$ nos experimentos (a) A, (b) B e (c) C e (d) densidade estimada pelo modelo de MCNABB \& BOERSMA (1993) (D est) em relação à densidade medida (DS) e a pressão aplicada (P, kPa). ${ }^{1} \mathrm{PD}=$ plantio direto desde o ano de 1989; PDË1 = plantio direto escarificado em fevereiro de 2002 e dezembro de 2004; PDE2 = plantio direto escarificado em dezembro de 2004; PDC1 = plantio direto com quatro passadas de máquina de $10 \mathrm{Mg}$ em dezembro do ano agrícola 2001/2002; PDC2 = plantio direto com quatro passadas de máquina de $10 \mathrm{Mg}$ em dezembro do ano agrícola 2002/2003; PDC3 = plantio direto com quatro passadas de máquina de $10 \mathrm{Mg}$ no em dezembro do ano agrícola 2001/2002 e 2002/2003.

relação aos demais tratamentos (PD e PDC1). Os resultados indicam que a utilização contínua do sistema PD poderá compactar camadas do solo, corroborando afirmações de STONE et al. (2002) e TORMENA et al. (1998).

A Ds e o IC foram os parâmetros mais sensíveis e consistentes para detectar alterações na estrutura desse solo. A compactação pode modificar a estrutura, a forma e a continuidade dos poros, reduzindo principalmente a porosidade do solo, com reflexos negativos na produtividade agrícola. A compactação é dependente do teor de matéria orgânica, umidade, mineralogia do solo, tipo, quantidade e duração da pressão aplicada (VOORHEES, 1992), dimensões e pressão de inflação dos pneus (SOANE et al., 1981). Diferentes valores de pressão pneumática têm sido recomendados (ALAKUKKU et al., 2003). Sugere-se que valores de pressão superiores a $153 \mathrm{kPa}$ (Figuras $2 \mathrm{a}, 2 \mathrm{~b}$ e $2 \mathrm{c}$ ) podem implicar condições favoráveis ao tráfego e inadequadas para o crescimento das raízes nessa área. Contudo, HORN \& FLEIGE (2003) consideram esse valor extremamente alto. Por outro lado, KANALI et al. (1997) indicam que a compactação pode ser crítica ao desenvolvimento das culturas a 
valores superiores a $121,5 \mathrm{kPa}$ para solos franco arenosos, com umidade e densidade média, respectivamente, de $0,23 \mathrm{~g} \mathrm{~kg}^{-1}$ e $1,40 \mathrm{Mg} \mathrm{m}^{-3}$. Por outro lado, SOANE et al. (1981) indicaram pressões inferiores a $200 \mathrm{kPa}$ e, preferencialmente, a 100kPa para um efetivo controle e redução da compactação do solo. Observouse que o modelo de MCNABB \& BOERSMA (1993) explicou significativamente a densidade do solo nos sistemas de manejo avaliados (Tabela 2). A partir dos valores médios, plotou-se a relação entre a densidade medida e a estimada (Ds est) a cada pressão aplicada (P) (Figura 2d). O modelo é de grande utilidade, uma vez que a densidade após o solo sofrer uma pressão pode ser estimada a partir do conhecimento da densidade inicial e das pressões a que o solo será submetido. A inclusão dos valores da pressão de preconsolidação no modelo possibilita determinar o valor de densidade que poderá causar a compactação adicional. Além disso, pode-se prever a pressão a que o solo foi submetido, conhecendo-se a densidade inicial e a obtida quando uma pressão é exercida sob um determinado tipo de solo (Figura 2d). Pelo conhecimento da pressão de preconsolidação, pode-se monitorar o tipo de tráfego, de maquinário e sistema a ser implantado em uma área agrícola. O modelo testado tem relevância na estimativa do grau de degradação estrutural do solo e como base de decisão na escolha de máquinas agrícolas a serem utilizadas.

\section{CONCLUSÕES}

No sistema de plantio direto sob compactação adicional, foram obtidos maiores e menores valores, respectivamente, de densidade e de índice de compressão do solo. A densidade e o índice de compressão do solo são parâmetros consistentes e sensíveis para detectar alterações na estrutura do solo. O modelo não-linear (MCNABB \& BOERSMA, 1993) é uma ferramenta potencial para a determinação da densidade do solo sob plantio direto, a partir da densidade inicial e das pressões aplicadas ao solo.

\section{REFERÊNCIAS}

ALAKUKKU, L. et al. Prevention strategies for field trafficinduced subsoil compaction: a review. Part 1. Machine/soil interactions. Soil and Tillage Research, v.73, p.145-160, 2003.

ALEXANDROU, A.; EARL, R. The relationship among the pre-compaction stress, volumetric water content and initial dry bulk density of soil. Journal of Agricultural Engeneering Research, v.71, p.75-80, 1998.

BLAKE, G.R.; HARTGE, K.H. Bulk density. In: KLUTE, A.
(Ed). Methods of soil analysis: physical and mineralogical methods. 2.ed. Madison: American Society of Agronomy, Soil Science Society of America, 1986. Cap.13, p.363-375.

CASAGRANDE, A. The determination of the pre-consolidation load and its practical significance. In: INTERNATIONAL CONFERENCE ON SOIL MECHANICS AND FOUNDATION ENGINEERING, D-34, 1936, Cambridge. Proceedings... Cambridge: MA Harvard University, 1936. p.60-64.

COLlares, G.L. Compactação em Latossolos e Argissolo em relação com parâmetros de solo e de plantas. 2005. 106f. Tese (Doutorado em Ciência do Solo) - Curso de Pósgraduação em Ciência do Solo, Universidade Federal de Santa Maria.

DIAS JUNIOR, M.S.; PIERCE, F.J. A simple procedure for estimating preconsolidation pressure from soil compression curves. Soil Technology, v.8, p.139-151, 1995.

EMPRESA BRASILEIRA DE PESQUISA AGROPECUÁRIA. Centro Nacional de Pesquisa de Solos. Sistema Brasileiro de Classificação de Solos. Brasília: Embrapa produção de informação, Rio de Janeiro: Embrapa Solos, 1999. 412p.

GUPTA, S.C.; ALLMARAS, R.R. Models to assess the susceptibility of soils to excessive compaction. Advances in Soil Science, v.6, p.5-10, 1987.

HORN, R.; FLEIGE, H. A method for assessing the impact of load on mechanical stability and on physical properties of soils. Soil and Tillage Research, v.73, p.89-99, 2003.

IMHOFF, S. et al. Susceptibility to compaction, load support capacity and soil compressibility of Hapludox. Soil Science Society of America Journal, v.68, p.17-24, 2004.

KANALI, C.L. et al. The use of soil compaction levels in the selection of the field-safe sugarcane transport vehicles. Journal of Terramechanics, v.34, p.127-140, 1997.

KONDO, M.K. Compressibilidade de três Latossolos sob diferentes usos. 1998. 95f. Dissertação (Mestrado em Agronomia) - Curso de Pós-graduação em Agronomia, Universidade Federal de Lavras.

KONDO, M.K.; DIAS JUNIOR, M.S. Compressibilidade de três Latossolos em função da umidade e uso. Revista Brasileira de Ciência do Solo, v.23, n.2, p.211-218, 1999.

KLUTE, A. Water retention: laboratory methods. In: KLUTE, A. (Ed). Methods of soil analysis: physical and mineralogical methods. 2.ed. Madison: American Society of Agronomy, 1986. Cap.26, p.635-660.

LARSON, W.E. et al. Compression of agricultural soils from eight soil orders. Soil Science Society of America Journal, v.44, n.3, p.450-457, 1980.

LEBERT, M.; HORN, R. A method to predict the mechanical strength of agricultural soils. Soil and Tillage Research, v.19, n.2/3, p.274-286, 1991.

LIMA, C.L.R. et al. Heterogeneidade da compactação de um Latossolo-Vermelho Amarelo sob pomar de laranja. Revista 
Brasileira de Ciência do Solo, v.28, p.409-414, 2004. MACNABB, D.H.; BOERSMA, L. Evaluation of the relationship between compressibility and shear strength of Andisols. Soil Science Society of America Journal, v.57, p.923-929, 1993.

MORENO, J.A. Clima do Rio Grande do Sul. Porto Alegre: Secretaria da Agricultura, 1961. 42p.

MOSADDEGHI, M.R. et al. Pre-compression stress and its relation with the physical and mechanical properties of a structurally unstable soil in central Iran. Soil and Tillage Research, v.70, p.53-64, 2003.

REINERT, D.J. et al. Compress - software e proposta de modelo para descrever a compressibilidade de solos e seus parâmetros. In: CONGRESSO BRASILEIRO DE CIÊNCIA DO SOLO, 29., 2003, Ribeirão Preto, SP. Anais... Ribeirão Preto: SBCS, 2003. CD-ROOM.

SALIRE, E.V. et al. Compression of intact subsoils under shortduration loading. Soil and Tillage Research, v.31, p.235248, 1994.

SECCO, D. et al. Produtividade de soja e propriedades físicas de um Latossolo submetido a sistemas de manejo e compactação. Revista Brasileira de Ciência do Solo, v.28, p.797-804, 2004.

SILVA, V.R. et al. Susceptibilidade à compactação de um Latossolo Vermelho Escuro e de um Podzólico Vermelho Amarelo. Revista Brasileira de Ciência do Solo, v.24, n.2, p.239-249, 2000.

SILVA, V.R. et al. Fatores controladores da compressibilidade de um Argissolo Vermelho Amarelo distrófico arênico e de um Latossolo Vermelho distrófico típico. I - Estado inicial de compactação. Revista Brasileira de Ciência do Solo, v.26, p.1-8, 2002a.
SILVA, V.R. et al. Fatores controladores da compressibilidade de um Argissolo Vermelho Amarelo distrófico arênico e de um Latossolo Vermelho distrófico típico. II - Grau de saturação em água. Revista Brasileira de Ciência do Solo, v.26, p.9-15, 2002 b.

SMITH, C.W. et al. Assessing the compaction susceptibility of South African forestry soils. II. Soil properties affecting compactibility and compressibility. Soil and Tillage Research, v.43, p.335-354, 1997.

SOANE, B.D. et al. Compaction by agricultural vehicles: a review II. Compaction under tyres and other running gear. Soil and Tillage Research, v.1, p.373-400, 1981.

SOANE, B.D. Process of soil compaction under vehicular traffic and means of alleviating it. In: LAL, R. et al. (Ed). Land clearing and development in the tropics. Rotterdam: Balkema, 1986. p.265-297.

STONE, L.F. et al. Compactação do solo na cultura do feijoeiro. I: efeitos nas propriedades físico-hídricas do solo. Revista Brasileira de Engenharia Agrícola e Ambiental, v.6, n.2, p.207-212, 2002.

STRECK, C.A. Compactação do solo e seus efeitos no desenvolvimento radicular e produtividade da cultura do feijoeiro e da soja. 2003. 83f. Dissertação (Mestrado em Agronomia) - Curso de Pós-graduação em Agronomia, Universidade Federal de Santa Maria.

TORMENA, C.A. et al. Propriedades físicas do solo sob plantio direto influenciadas por calagem, preparo inicial e tráfego. Revista Brasileira de Ciência do Solo, v.22, p.301-309, 1998.

VOORHEES, W.B. Wheel-induced soil physical limitations to root growth. Advances in Soil Science, v.19, p.73-95, 1992. 